\title{
Synthesis and Thermal Performance of
}

\section{Microencapsulated Binary Carbonate Molten}

\author{
Salts for Solar Thermal Energy Storage
}

\author{
Supporting Information \\ Jaejun Lee and Byeongnam Jo ${ }^{a^{*}}$ \\ ${ }^{a}$ Department of Mechanical Engineering, Ajou University, Suwon 16499, Korea \\ ${ }^{*}$ Corresponding author e-mail: jo798@ajou.ac.kr
}
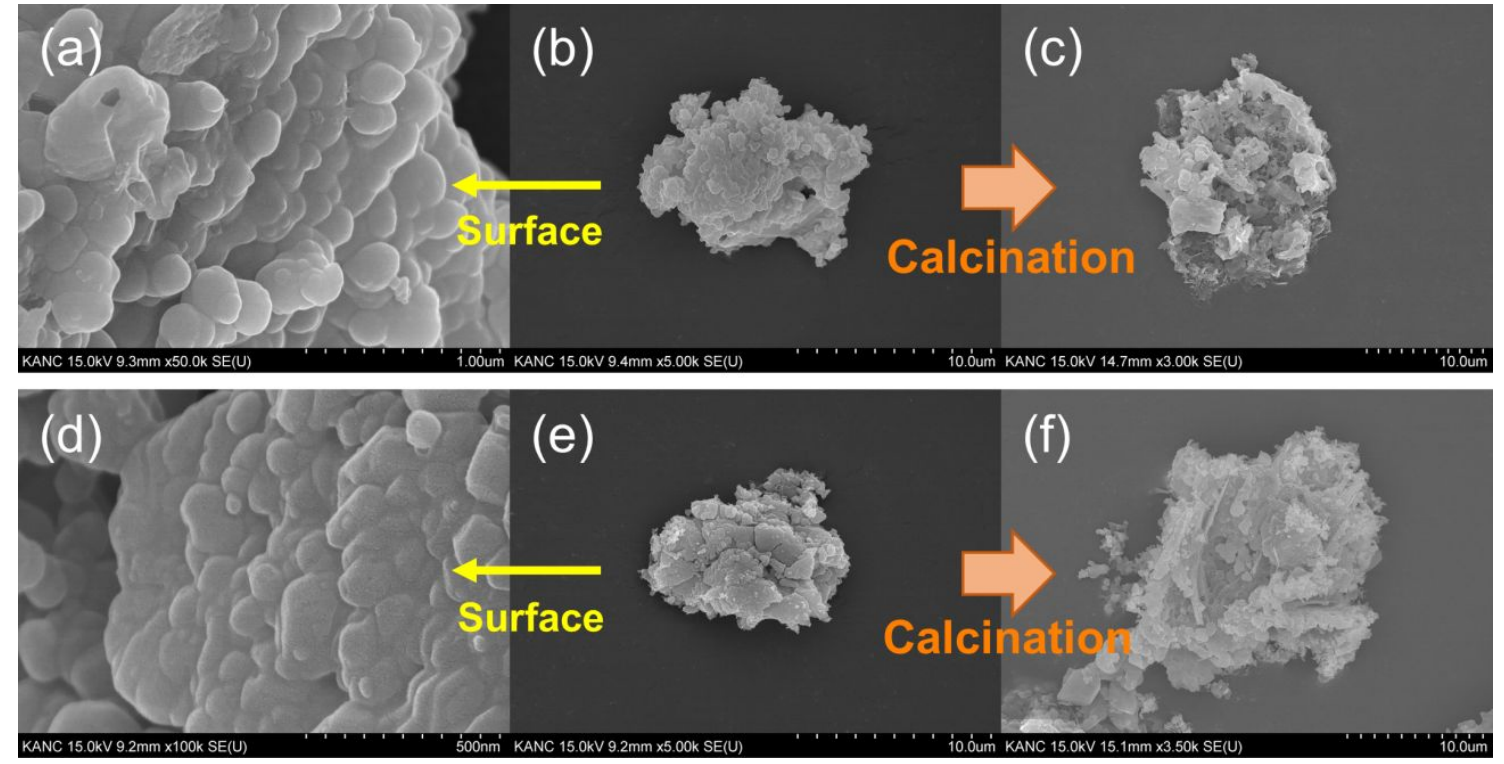

Figure S1. Secondary electron images of S-1 (a, b, c) and S-2 (d, e, f).

Figure S1 presents SEM images of the microcapsules (S-1 and S-2). Figure S1(a) to S1(c) was obtained from S-1 and Figure S1(d) to S1(e) was obtained from S-2. As shown in Figure S1(a) and S1(b), a smooth silica shell is formed under strong base-catalyzed conditions (high $\mathrm{NH}_{4} \mathrm{OH}$ concentration) on 
the jagged surface of the binary carbonate salt particles. However, a broken silica shell can be observed after calcination at $400{ }^{\circ} \mathrm{C}$ for $3 \mathrm{~h}$, as shown in Figure $\mathrm{S} 1$ (c). Figure. S1(d) to $\mathrm{S} 1(\mathrm{e})$ presents the shell surface and overall morphology of the S-2 microcapsules. Similar to S-1, the silica shell of S-2 also has a smooth surface. Additionally, although it is not as severe, the silica shell of the S-2 microcapsules also shows damage after calcination (Figure S1(f)). Based on our SEM analysis of the S-1 and S-2 samples, we can conclude that silica shells synthesized in ethanol and isopropanol are vulnerable at elevated temperatures up to $400{ }^{\circ} \mathrm{C}$, which is lower than the melting temperature of the core salt.

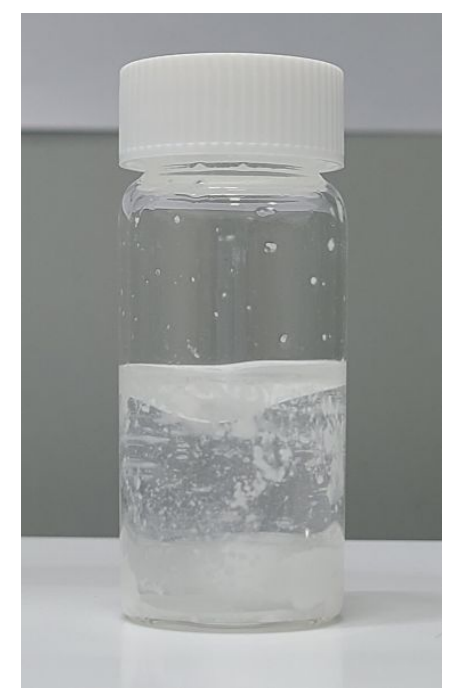

Figure S2. Image of eutectic carbonate salt agglomerated in a propylene carbonate solvent.

Propylene carbonate (99\%, Junsei Chemical) was tested as a solvent for the sol-gel process in this study. However, the eutectic carbonate salt particles were not dispersed and exhibited slurry-like behaviors in the propylene carbonate, as shown in Figure S2. This can be attributed to the high dielectric constant of propylene carbonate. Therefore, subsequent shell synthesis was not performed using this solvent. 